Umweltpolitik aus der Perspektive der Neuen Außenwirtschaftstheorie

\title{
Öko-Dumping vs. First-Mover-Vorteil
}

\author{
In der öffentlichen Debatte wird oft befürchtet, daß einer strikten Umwelt- \\ politik ihre Auswirkungen auf die internationale Wettbewerbsfähigkeit \\ entgegenstehen. Wöhrend die traditionelle Außenwirtschaftstheorie hierfür \\ keine Grundlage biefet, liefern nevere Theorieansätze dieser Befürchtung mehr \\ Nahrung. Zugleich erlauben diese aber auch die Analyse der Bedingungen, \\ unter denen positive Wettbewerbseffekte entstehen können.
}

$\mathrm{D}$ e Koalitionsvereinbarung zwischen der Sozialdemokratischen Partei Deutschlands und Bündnis 90/ Die Grünen hält fest: „Wir streben eine größere Harmonisierung der Umweltvorschriften in der Europäischen Union auf hohem Niveau an. Wir wollen internationale Vereinbarungen gegen Umweltdumping." Aus Sicht der neoklassischen Außenhandelstheorie ist diese Forderung nicht nachvollziehbar. ÖkoDumping und umweltbedingte Kapitalflucht stellen aus ihrer Sicht kein politikrelevantes Problem dar, zumindest solange keine grenzüberschreitenden Umweltschäden vorliegen. Handelsströme und Kapitalflïsse reagieren in diesem Modellrahmen zwar sehr sensitiv auf Differenzen der Umweltpolitiken und es ergibt sich eine entsprechende Spezialisierung der Länder, aber diese internationale Arbeitsteilung wird als wohlfahrtsökonomisch positiv eingeschätzt. Ähnliches gilt im Prinzip für umweltbedingte Kapitalflüsse bzw. deren Veränderung. Allerdings unterliegt die traditionelle Theorie zunehmender Kritik. Die insbesondere mit dem Namen Paul Krugman verbundene sogenannte Neue Außenwirtschaftsheorie betont vor allem die Vernachlässigung steigender Skalenerträge und des dadurch implizierten unvollkommenen Wettbewerbs. Die Grundlage bildet die empirische Beobachtung, daß ein großer Teil des Welthandels inzwischen auf oligopolistischen Märkten bzw. innerhalb von Industrien stattindet. Die Neue Außenwirtschaftstheorie verbindet daher industrieökonomische Erkenntnisse mit der Theorie des internationalen Handels (1). Viele von ihr behandelte Aspekte sind a priori auch für die umweltpolitische Debatte von Interesse, z.B. der Einbezug von Unternehmen als strategischen Akteuren, Produktdifferenzierung und die Bedeutung von Handels- und Transportkosten als Puffer für politische Spielräume. Auch sind dies die Outputmenge, wählen. In Anlehnung an das Modell strategischer Handelspolitik lassen sich niedrigere Umweltstandards als kostensenkende Subvention für das inländische Unternehmen interpretieren. Sie führen ceteris paribus zu einer Steigerung von Output und Gewinnen des inländischen Oligopolisten auf Kosten seines Konkurrenten und einem generellen Outputanstieg (3).

Vergleicht man die Wirkung von Umweltpolitik im Oligopol mit der bei vollkommener Konkurrenz, so ist festzustellen, daß eine Kostensteigerung durch Umweltpolitik hier in der Regel nicht zum Marktaustritt der betroffenen Firma, sondern nur zu einer Verschiebung der Marktanteile führt, solange sie nicht die Oligopolrenten völlig verschwinden läßt. Gerade aus der Existenz dieser Renten ergeben sich allerdings wohlfahrtsökonomisch Rückwirkungen für die politische Wahl der Umweltstandards. Unter bestimmten Annahmen besteht selbst bei rein nationalen Umweltbeeinträchtigungen ein Anreiz für die Regierung, niedrigere Standards zu setzen als umweltpolitisch optimal, um den Gewinn von Marktanteilen für die inländische Firma zu bewirken und damit Renten umzulenken, solange die Wohlfahrtsverluste aus dem höheren Umweltschaden hierdurch (über)kompensiert werden.

Allerdings gilt dieser Anreiz zu Öko-Dumping symmetrisch für beide Regierungen. In dynamischer Betrachtung kommt es in einem nichtkooperativen Setting zu einem schädlichen Wettlauf nach unten, in dem die Regierungen jeweils mit einer weiteren Lockerung der Umweltpolitik auf entsprechendes Verhalten der anderen reagieren. Dieses qualitative Ergebnis ist aber entscheidend an eine Reihe von Voraussetzungen gebunden.

Wichtig ist hier zum einen, daß andere handelspolitische Instrumente wie Zölle und Subventionen nur beschränkt zur Verfügung stehen, denn aufgrund der hervorgerufenen Umweltschäden hat strategische Umweltpolitik eindeutig Second-Best-Charakter. Zum zweiten hängt die Plausibilität des Ergebnisses von zentralen Charakteristika der betroffenen Industrien $a b$. Wichtig sind hier ein hoher Konzentrationsgrad der inländischen Industrie (also ein enges Oligopol) und die Marktform des oligopolistischen Wettbewerbs. Das Ergebnis gilt so nur für Mengenwettbewerb (sogenanntes Cournot-Verhalten); bei Preiswettbewerb können (aber müssen nicht) die Ergebnisse diametral verschieden 
ausfallen. In der Industrieökonomik hat sich gezeigt, daß bei homogenen Produkten die Cournot-Ergebnisse besser mit dem beobachtbaren Unternehmensverhalten in Einklang $\mathrm{zu}$ bringen sind, insbesondere wenn (fixe) Investitionskosten hoch und Kapazitätsgrenzen von Bedeutung sind.

Allerdings werden diese Ergebnisse in einem vereinfachten Setting abgeleitet, das weitere strategische Wahlmöglichkeiten des Unternehmens selbst wie Produktdifferenzierung, Innovationen und die Standortwahl nicht berïcksichtigt. Die Erstgenannten bilden z.B. eine wichtige Grundlage für die sogenannte Porter-Hypothese, die sogar Wettbewerbsvorteile durch strikte Umweltpolitik postuliert (4). Wie verändern sich daher die Ergebnisse, wenn diese Faktoren im Rahmen von etwas komplexeren Modellen berücksichtigt werden?

\section{Umweltpolitik und Standortwahl}

Ein Großteil der Diskussion um außenwirtschaftliche Grenzen der Umweltpolitik läßt sich gerade an der Befürchtung festmachen, Unternehmen könnten aufgrund „strengerer“ Umweltpolitik in andere Länder abwandern. In einem typischen Modellrahmen von Markusen/ Morey/ Olewiler, der dies zuläßt, wird die Marktstruktur selbst zu einer endogenen Variable. Zahl und Standort der Produktionsanlagen hängen jetzt nicht nur von ,technologischen“ Faktoren wie firmen- und anlagespezifischen Fixkosten und Transportkosten, sondern auch von der Striktheit der Umweltpolitik ab (5).

Damit wird abbildbar, daß Umweltpolitik einen diskontinuierlichen Einfluß auf die Wettbewerbsbedingungen hat; (nur) bei Überschreitung bestimmter Schwellenwerte, die u.a. von der Mobilität des Sektors abhängen, fiihrt sie zu einer Veränderung der Marktstruktur und somit zu Firmenverlagerungen bzw. zum Marktaustritt. Diese sind allerdings aufgrund der verlorengehenden Renten dann wohlfahrtsrelevant. Dieser Niveaueffekt wird durch Agglomerationsvorteile und Spillovers zwischen Industrien noch verstärkt. Weiterhin hängen die Wohlfahrtswirkungen stark davon ab, ob die Kosten der Umweltregulierung auf die fixen oder die variablen Kosten fallen. Im ersten Fall kann die verstärkte Nutzung von Skalenvorteilen durch die induzierte Rationalisierung ausgleichend wirken, im zweiten Fall gilt das Gegenteil (6).

Betrachtet man wiederum die entstehende Dynamik der Umweltpolitik, so ergibt sich in ver- schiedenen Modellvarianten, daß sowohl ein Race to the Bottom als auch ein Race to the Top (der „Not-in-my-Backyard-Fall“) möglich ist (7). Ein Wettlauf nach unten ist jetzt aber für eine größere Zahl von Marktstrukturen wahrscheinlich, solange der Umfang der produktionsbedingten Umweltschäden nicht zu groß ist. Er ist jedoch nur rational, wenn ,first best"Maßnahmen wie Ansiedelungssubventionen nicht verfügbar sind.

\section{Öko-Innovation und Produkt- differenzierung als Königsweg?}

Auch Produktdifferenzierung ist entgegen manchen Vermutungen kein genereller Ausweg aus dem Weltmarktkonkurrenz-Dilemma. Dies wird unisono von unterschiedlichen Modelltypen gezeigt. Selbst bei der Nutzung von ökologischen Produktstandards als strategisches Differenzierungsinstrument im Oligopol sind ökologische Mindeststandards nur unter spezifischen Annahmen ein geeignetes politisches Instrument; dabei wird in den bisher vorliegenden Modellen über die ökologische Bewertung ihrer Höhe wenig ausgesagt.

Auch Investitionsentscheidungen können durch ihren strategischen Charakter den Verlauf des Wettbewerbs auf dem Produktmarkt beeinflussen. Umweltpolitisch zentral sind hier Innovationen bzw. FuE-Entscheidungen. Entgegen der Vermutung der Porter-Hypothese läßt sich aber selbst hier kein robustes positives Ergebnis ableiten; die Ansätze im Rahmen der Neuen Außenwirtschaftstheorie stïtzen selbst bei FirstMover-Vorteilen die Koinzidenz von Umweltpolitik und Wettbewerbsvorteilen nur begrenzt (8). Allerdings lassen sich nicht alle Einflußfaktoren, die hierbei eine Rolle spielen können, im Rahmen der Modelle der Neuen Außenwirtschaftstheorie befriedigend analysieren; insbesondere sind hier dynamische Aspekte von Innovationen (z.B. der ,early home demand effect") zu nennen. Anders als bei Porter wird zudem vollständige Information vorausgesetzt. Auch ist die Art des Einbezugs ökologischer Aspekte unbefriedigend. Die Umweltbeeinträchtigung wird als ein Element unter vielen gegen die anderen Aspekte abgewogen und bei Bedarf aus strategischen Gründen instrumentalisiert. So sehr daher die vorgestellten Ansätze ökologisch noch viele Leerstellen aufweisen, so kann die Neue Außenwirtschaftstheorie doch zur Erklärung der Diskrepanzen zwischen normativem Anspruch ökologisch-orientierter Theorie und beobachtbarer ökonomischer Praxis ein Stiick weit beitragen, da sie es erlaubt, außenwirtschaftliche Restriktionen der Umweltpolitik zu thematisieren, und zugleich die Möglichkeit gegenläufiger Effekte im Blick zu behalten.

\section{- Politische Konsequenzen}

Politisch relevant sind die abgeleiteten Ergebnisse insbesondere dann, wenn nur wenig andere politische Instrumente als wettbewerbsbezogene Ressourcen genutzt werden können. Dies scheint in der Realität angesichts zunehmender weltwirtschaftlicher Integration immer mehr der Fall zu sein, als Beispiele seien nur EU und WTO genannt. Auch ist die Plausibilität einer Politik des Öko-Dumping an hohe informationelle Anforderungen gebunden. Um entsprechend agieren zu können, muß die Regierung sowohl die genaue Industriestruktur kennen als auch in der Lage sein, ein glaubwürdiges Subventionssignal zu setzen. Im Politikprozeß wird dies vor allem dann brisant, wenn es die Politik mit rentenerzielenden oligopolistischen Industrien $\mathrm{zu}$ tun hat, die diese Renten auch fiur Einflußnahme auf den politischen Prozeß verwenden können. Gerade wenn die Geltung des Rent-ShiftingArguments fraglich ist oder Unsicherheit über die konkreten Wirkungen besteht, kann es von Seiten der ProduzentInnen politisch benutzt werden.

Eine kritische Einschätzung der Politikrelevanz, wie sie häufig vorgenommen wird, erscheint also nicht völlig unberechtigt. Allerdings reicht es nicht aus, die Relevanz schon mit dem Argument abzulehnen, daß die Umweltregulierungen in vielen Industrieländern ähnlich seien. Dies ließe sich nämlich auch als Folge eines ÖkoDumping-Dilemma deuten.

Wie könnte der Öko-Dumping-Gefahr politisch begegnet werden? Zum einen wird z.B. vorgeschlagen, mittels Umweltsteuern und daraus finanzierten Energiesparsubventionen einen ökologisch vorteilhafteren internationalen Wettbewerb zu initiieren (9). Zum anderen können kooperative Lösungen selbst bei lokalen Umweltschäden prinzipiell sinnvoll sein, um Öko-Dumping zu verhindern oder zumindest einzugrenzen. Diese im konventionellen Theorierahmen nicht ableitbare Schlußfolgerung gewinnt besondere Relevanz, wenn es um die Harmonisierung der Umweltpolitiken in der Europäischen Union geht. Daher scheint eine empirische Überprüfung, die bisher noch weitgehend fehlt, auf jeden Fall notwendig. Ein 
explorativer Versuch, den skizzierten theoretischen Rahmen auf die Frage der Harmonisierung der Umweltpolitik im Rahmen der EUOsterweiterung anzuwenden, stößt zwar auf eine Reihe von empirischen Problemen, verdeutlicht aber zugleich Möglichkeiten und Grenzen der Ansätze (10).

\section{Anmerkungen}

(1) Vgl. für einen Überblick Helpman, Elhanan/ Paul Krugman: Market Structure and Foreign Trade. Cambridge MA/ London 1985, MIT Press sowie dies.: Trade Policy and Market Structure. Cumbridge MA/ London 1989, MIT Press. (2) Vgl. für eine ausführliche Analyse Nill, Jan: Zwischen Öko-Dumping und First-Mover-Vorteilen. Die Perspektive der Neuen Außenwirtschaftstheorie auf Umweltpolitik und eine explorative Anwendung auf die EU-Osterweiterung. IÖWSchriftenreihe 139/99, Berlin.

(3) Vgl. grundlegend Barrett, Scott: Strategic Environmental Policy and International Trade. Journal of Public Economics, Vol. 54 (1994), S. 325-338 sowie für eine Analy- se der meisten im folgenden skizzierten Aspekte Rauscher, Michael: International Trade, Factor Movements, and the Environment. Oxford 1997, Clarendon Press.

(4) Porter, Michael E./ Claas van der Linde: Toward a New Conception of the Environment-Competitiveness Relationship. Journal of Economic Perspectives, Vol. 9 (1995), №. 4, S. 97-118.

(5) Vgl. grundlegend Markusen, James R./ Edward R. Morey/ Nancy D. Olewiler: Environmental Policy when Market Structure and Plant Locations Are Endogenous. Journal of Environmental Economics and Management, Vol. 24 (1993), S. 69-86.

(6) Vgl. Markusen, James R.: Costly Pollution Abatement, Competitiveness and Plant Location Decisions. Resource and Energy Economics, Vol. 19 (1997), S. 299-320.

(7) Vgl. z.B. Hoel, Michael: Environmental Policy with Endogenous Plant Locations. Scandinavian Journal of Economics, Vol. 99 (1997), S. 241-259.

(8) Vgl. z.B. Ulph, Alistair/ David Ulph: Trade, Strategic Innovation and Strategic Environmental Policy. A General Analysis. In: Carraro, Carlo/ Yannis Katsoulacos/ Anastasios Xepapadeas (Eds.): Environmental Policy and Market
Structure. Dordrecht et al. 1996, Kluwer Academic Publishers, S. 181-208 und

Simpson, R. David/ Robert L. Bradford: Taxing Variable Cost: Environmental Regulation as Industrial Policy. Journal of Environmental Economics and Management, Vol. 30 (1996), S. 282-300.

(9) Vgl. z.B. Ziesemer, Thomas: Reconciling Environmental Policy with Employment, International Competitiveness and Participation Requirements. MERIT-Paper 2/1995-022, Maastricht, Maastricht Economic Research Institute on Innovation and Technology.

(10) Vgl. hierzu Nill a.a.0.

\section{Der Autor}

Jan Nill ist wissenschaflicher Milarbeiter an der Fachhochschule für Wirtschatt Berlin und am IÖW. Kontakt: IÖW, Poisdamer Str. 105, 10785 Berlin. Tel. 030/884594-0, Fax 030/8825439,

E-mail: Jan.Nill@ioew.de

\section{ZEITSCHRIFTENSCHAU}

\section{Ecological Economics, Vol. 28/2}

P. Söderbaum: Values, ideology and politics in ecological economics

E.H. Bulte, G.C. van Kooten: Economic efficiency, resource conservation and the ivory trade ban

T.C. Krehbiel, R.F. Gorman, O.H. Erekson, O.L. Loucks, P.C. Johnson: Advancing ecology and economics through a business-science synthesis

K. Rennings, H. Koschel, K.L. Brockmann, I. Kühn: A regulatory framework for a policy of sustainability: lessons from the neo-liberal school

P.A. Lawn, R.D. Sanders: Has Australia surpassed its optimal macroeconomic scale? Finding out with the aid of 'benefit' and 'cost' accounts and a sustainable net benefit index

B.E. Castañeda: An index of sustainable economic welfare (ISEW) for Chile

J. Agras, D. Chopman: A dynamic approach to the Environmental Kuznets Curve hypothesis

\section{Ecological Economics, Vol. 28/3}

J. Barry, J. Proops: Seeking sustainability discourses with $Q$ methodology

M. Cogoy: The consumer as a social and environmental actor

I. Røpke: The dynamics of willingness to consume

T. Jackson, N. Marks: Consumption, sustainable welfare and human needs - with reference to UK expenditure palterns between 1954 and 1994

F.E. Prettenthaler, K.W. Steininger:

From ownership to service use lifestyle:

the potential of car sharing

\section{Zeitschrift für}

\section{Umweltpolitik \& Umweltrecht $1 / 99$}

J. Blazejczak, D. Edler, J. Hemmelskamp, M. Jänicke:

Umweltpolitik und Innovation: Politikmuster und Innovationswirkungen im internationalen Vergleich

J. Hemmelskamp: Der Einfluß der Umwelipolitik aul das Innovationsverhalten - eine ökonometrische Untersuchung

M. von Houff, D. Solbach: Perspektiven integrierter

Umweltschutztechnologie in der Bundesrepublik

Deutschland

K.W. Zimmermann, E. Zirkmann: Das Technologie-Puzzle: Umwelisparende Anpassung in Unternehmen

II. Pflüger: Globalisierung und Nachhaltigkeit

\section{Zeitschrift für angewandte Umweltforschung $1 / 99$}

Umweltdiskussion: Beratung in der Umweltpolitik durch Sachverständigengremien. Mit Beiträgen von H. Wiggering, M. Hüther und M. Schulz-Baldes

H. Welsch: C02-Sieuern, Beschäftigung und funktionale Einkommensverteilung

E. Gawel: Das beschleunigte Immissionsschutzrechı des Bundes: Eine ökonomische Evaluierung der Beschleunigungsnormen im BIMSchG nach der 5. Novelle B. Klaver: Was ist Nachhaltigkeit und wie kann man eine nachhaltige Entwicklung erreichen?

E. Wickop: Qualitätsziele für eine nachhaltige Stadtentwicklung

B. Citlak, M. Kreyenfeld: Wahrnehmung von Umweltrisiken - Empirische Ergebnisse für die Bundesrepublik Deutschland
R. Weiland: Ökologisierte Subventionspolitik? - Ansatz und Grenzen ökologisch motivierter Subventionen in der aktuellen Umweltpolitik

\section{UmweltWirtschaftsForum $1 / 99$}

Schwerpunktthema: Organisation des betrieblichen Umwelischutzes. Mit Beiträgen von R. Artischewski, J. Bover, R. Schwaderlapp und R. Antes

G. Jürgens, J. von Steinaecker: Integrierter Umweltschuiz als Ziel des Umweltmanagements - Projekt bei Trumpt $\mathrm{GmbH}+\mathrm{C}_{0}$.

U. Kirschten: Öko-Controlling bei KUNERT T. Claes, P. Böggemann, R. Pfriem: Umweltkostenrechnung als Teil der betrieblichen Kostenrechnung - Projekt bei der Deutschen Bahn AG

F. Nehm: Lebenszyklus-Analyse am Beispiel der Papierkette durch multinationale Kooperationen H. Kottmann, J. Clausen, T. Loew: Umweltkennzahlen für den betrieblichen Verbesserungsproze $\mathbb{B}$ D. FeB, R.J. Ostendorf: Untersuchung von Öko-Controlling-Ansätzen auf ihre Anwendbarkeit in KMU H.-K. Arndt: Beurteilung betrieblicher Umwelischutzsoftware C. Empacher, T. Kluge: Die Integration der sozialen Dimension in eine nachhaltige Unternehmenspolitik C. Zanger, J. Drengner, H. Gaus: Konsumentenakzeptanz von Nutzungsdaververlängerung und -intensivierung

\section{Ambio, Vol. 26/2}

E.B. Barbier, T.F. Homer-Dixon: Resource Scarcily and Innovation: Can Poor Countries Attain Endogenous Growth? F. D'Evie, J. Taylor: Greenhouse Gas Emission Abatement: Equitable Burden Sharing 
(c) 20I0 Authors; licensee IÖW and oekom verlag. This is an article distributed under the terms of the Creative Commons Attribution Non-Commercial No Derivates License (http://creativecommons.org/licenses/by-nc-nd/3.o/), which permits unrestricted use, distribution, and reproduction in any medium, provided the original work is properly cited. 